\title{
ZNF582 Methylation Predicts Better Radiotherapy Response of Cervical Cancer and ZNF582 Overexpression Reduces Radiosensitivity by Arresting the Cell Cycle in S Phase
}

\author{
Nayiyuan Wu \\ Hunan Cancer Hospital \\ Xiaoyun Zhang \\ Hunan cancer hospital \\ Miaochen Zhu \\ Hunan cancer hospital \\ Chao Fang \\ Hunan Cancer Hospital \\ Xiaoting Liu \\ The second people's hospital of hunan province \\ Ying Wang \\ Hunan cancer hospital
}

He Li

Hunan cancer hospital

Siye Liu

Hunan cancer hospital

Hong Ting

Hunan Cancer Hospital

Chongzhen Qin

Zhengzhou University First Affiliated Hospital

Qianjin Liao

Hunan Cancer Hospital

JingTing Cai

Hunan Cancer Hospital

Jing Wang ( $\triangle$ wangjing0081@hnca.org.cn )

Hunan Cancer Hospital https://orcid.org/0000-0001-7683-6259

Research Article 
Keywords: Cervical cancer, ZNF582, cell cycle, radiosensitivity, methylation

Posted Date: October 21st, 2021

DOI: https://doi.org/10.21203/rs.3.rs-990658/v1

License: (c) (1) This work is licensed under a Creative Commons Attribution 4.0 International License. Read Full License 


\section{Abstract}

Purpose: Our previous studies identified ZNF582 methylation $\left(Z N F 582^{m}\right)$ level as a useful biomarker for cervical cancer screening, detection, and prognosis. The purpose of this study is to investigate the relationship between $Z N F 582^{m}$ level and cervical cancer radiotherapy sensitivity and its underlying mechanism.

Patients and Methods: This was a prospective multicenter clinical study, included two independent cohorts locally advanced cervical cancer patients. Exfoliated cervical cancer cells were collected pretreatment and during treatment (after $24,30,36,48$, and 64 Gy) to test $Z N F 582^{m}$ level, radiotherapy response evaluated according to RECIST Version.

Results: In the first cohort, 22 cases achieved satisfied response, 28 exhibited modest response. Radiotherapy reduced $Z N F 582^{m}$ level among all patients. Baseline $Z N F 582^{m}$ was significantly higher in the satisfied response cases than in modest response cases, also, patients with high baseline ZNF582 ${ }^{m}$ (ZNF582 $\left.{ }^{m-h i g h}, \mathrm{n}=21\right)$ were more sensitive to radiotherapy than $Z N F 582^{m-l o w}$ patients $(\mathrm{n}=29)$, as evidenced by greater satisfied response rate (76.2\% vs. $20.7 \%)$. An independent cohort confirmed above results. The magnitude of $Z N F 582^{m}$ reduction was associated with a radiotherapeutic response, a subset of ZNF582 $2^{\text {m-low }}$ patients (5 of 28) exhibiting a transient increase in $Z N F 582^{m}$ demonstrated greater radiosensitivity than other ZNF582 ${ }^{m-l o w}$ patients. ZNF582 overexpression induced cell cycle arrest in $S$ phase.

Conclusion: High $Z N F 582^{m}$ level predicts better cervical cancer radiosensitivity, ZNF582 overexpression reduces radiosensitivity by cell cycle arrest.

\section{Introduction}

Cervical cancer is a major global health concern despite intensive screening and vaccination initiatives[1, 2]. More than $85 \%$ of new cases and $90 \%$ deaths occur in developing countries[3, 4]. In China, cervical cancer is still a huge burden with high incidence $(7.5 / 100,000)$ and mortality $(3.4 / 100,000)[5]$, and effective treatment remains a challenge as a high proportion of new cases in developing countries are already at an advanced stage (IB2 or more) at diagnosis[6].

Most advanced cervical cancer cases receive external beam radiotherapy and brachytherapy with or without chemotherapy as standard treatment[6-8]. Radiotherapy has been used as cancer treatment for more than a century, and with continued progress in precision delivery, the tumor-targeted dose has greatly increased. However, adverse reactions such as radiation vaginitis, cystitis, and proctitis still negatively influence quality of life[9]. These side effects may be exacerbated by radiotherapy resistance and resulting need for greater therapeutic doses. Indeed, resistance to radiotherapy accounts for most therapeutic failures in patients with cervical cancer[10,11]. These failures highlight the necessity of developing more personalized radiotherapy treatments for cervical cancer. Therefore, it is critical to 
identify reliable biomarkers predictive of treatment response, including predictors of radiotherapy resistance.

Zinc Finger Protein 582 (ZNF582) is located on chromosome 19 encodes a nuclear protein which contains one KRAB-A-B domain and nine zinc-finger motifs. The ZNF582 protein is predicted to be an intracellular protein transcription factor with five transcripts encoding five different proteins, and ZNF582 gene may also be involved in DNA damage response, proliferation, cell cycle control, and tumor transformation[12, 13]. In 2012, ZNF582 was found to be hypermethylated in cervical cancer tissues for the first time [14]. Our previous studies shown that ZNF582 was hypermethylated in cervical cancer[1519] and ZNF582 methylation-positive cervical adenocarcinoma patients demonstrated better prognosis[16]. We also found that ZNF582 methylation levels were reduced in concomitant chemoradiotherapy (CCRT) patients compared with that in non-CCRT patients [19]. While the relationship between ZNF582 methylation level and chemoradiotherapy sensitivity and its potential mechanism are unclear.

\section{Materials And Methods}

\section{Study design and patient cohort}

This was a prospective multicenter clinical study, including patients with a diagnosis of cervical cancer between October 2017 and May 2019. Inclusion criteria are as follows (1) patients with histologically confirmed cervical cancer, (2) patients with tumor staged IB1-IVa (FIGO 2009 definition), (3) patients who were not previously treated with any anti-cancer treatment, and (4) patients who underwent a pretreatment contrast-enhanced computed tomography (CT) scan. Exclusion criteria were (1) patients with a history of previous chemotherapy or radiotherapy, (2) patients with a diagnosis of other cancers, or (3) patients with distant metastatic disease (para-aortic nodes involvement was not included). Institutional ethics review board approval was acquired for this study and written informed consent was waived.

All patients were treated with image-guided external beam radiotherapy (EBRT) and brachytherapy (BT), to a total dose of 85-90 Gy (EQD2, equivalent dose in 2 Gy single dose fractions). EBRT was delivered in 1.8-2.0 Gy/fraction, to a range of 45-50 Gy, using a 3D conformal technique. BT boost was volumetrically planned and delivered as weekly high-dose-rate fractions of 8 Gy EQD2 each after 15 times of EBRT. Concurrent weekly chemotherapy with cisplatin $(40 \mathrm{mg} / \mathrm{m} 2)$ was delivered for 4-6 weeks or carboplatin (AUC = 2) when feasible. We proposed ZNF582 methylation performance in a two-stage group sequential design as described in the following text:

The first stage: cervical exfoliated cells were collected pre-treatment (0 Gy) and periodically during treatment (after 24, 30, 36, 48, and 64 Gy). All liquid-based cytology samples were clinician-collected using the Cytoprep Brush (Hospitex Diagnostics SRL, Sesto Fiorentino, Italy) and preserved in CytoFast Solution (Hospitex Diagnostics SRL, Sesto Fiorentino, Italy). The cells were centrifuged and stored in phosphate-buffered saline (PBS) at $-20^{\circ} \mathrm{C}$ from the day of collection. 
The response to radiotherapy was evaluated by two skilled radiologists according to Response Evaluation Criteria in Solid Tumors (RECIST) Version 1.1 with the following specific standards: 1. complete remission (CR), defined as disappearance of all target foci; 2 . partial response (PR), defined as a $\geq 30 \%$ reduction in total lesion diameter from baseline; 3 . progressive disease (PD), defined as a $\geq 20 \%$ increase in total lesion diameter from baseline; 4 . stable disease (SD), defined as an increase of $<20 \%$ or a reduction of $<30 \%$ in total lesion diameter from baseline. In this study, CR and PR were defined as satisfied response, SD as a modest response, and PD as non-response. ROC curve analysis was used to determine which M-index of the target gene ZNF582 would have the highest accuracy for discerning satisfied response from modest response and non-response.

The second stage, we confirmed the research result by an independent cohort at three centers (Hunan Cancer Hospital, Shandong Cancer Hospital, and Liaoning Cancer Hospital).

The study protocol was approved by the Institutional Review Board of Hunan Cancer Hospital. Informed consent was obtained from all participants in accordance with the Declaration of Helsinki.

\section{DNA preparation}

Genomic DNA (gDNA) was extracted from the collected cells using the QIAamp DNA Mini Kit (Qiagen $\mathrm{GmbH}$, Hilden, Germany). A BioSpec-nano spectrophotometer (Shimadzu Corporation, Tokyo, Japan) was used to quantify the amount of extracted DNA.

\section{DNA methylation tests}

For DNA methylation tests, quantitative methylation specific PCR (QMSP) using TaqMan-based technologies was performed using the Lightcycler LC480 real-time PCR system (Roche Applied Science, Penzberg, Germany). Briefly, 500 ng gDNA was subjected to bisulfite conversion using the EZ DNA Methylation-Gold ${ }^{\text {TM }}$ Kit (Zymo Research, CA, USA). The methylation levels of ZNF582 were then determined using the qPCR kit (Hongya Gene Technology Co., Ltd); the type II collagen gene (COL2A) was designed as the internal reference and tested with each specimen. The crossing point (Cp) value for COL2A, which is also the validity indicator of the test, should not be $>35$. For each sample, two $C p$ values were obtained: one from ZNF582 and another from COL2A. The DNA methylation level was assessed based on the methylation index (M-index) using the formula: 10,000 $\times 2$ [(Cp of COL2A) - (Cp of ZNF582)][16, 19].

\section{Establishment of ZNF852 overexpression cell line}

HeLa (CLS Cat\# 300194/p772_HeLa, RRID:CVCL_0030) cells were transfected with $20 \mu \mathrm{g}$ of ZNF582 expression adenoviral vector (Vigene Biosciences, Rockville, MD, USA; NM_144690) or control adenoviral vector (Vigene Biosciences, Rockville, MD, USA; pLent-GFP-Puro-CMV), screening of transferred cells with puromycin.

\section{Cell viability assay}

Cultured cells in the logarithmic growth phase were harvested using trypsin and seeded onto 96-well plates at 5000 cells/well. The culture medium was aspirated off and $100 \mu \mathrm{L}$ XTT solution (20-300-1000, 
Biological Industries) was added at the indicated times, followed by incubation for $3 \mathrm{~h}$. Optical density values were measured at $450 \mathrm{~nm}$ as an estimate of viable cell number using a microplate reader.

\section{Cell cycle analysis by flow cytometry}

Cells were collected, washed with cold PBS, fixed in $70 \%$ ethanol, and stored at $4^{\circ} \mathrm{C}$ overnight. The fixed cells were washed with PBS and stained with $500 \mu \mathrm{L}$ propidium iodide (PI; BD Biosciences, Franklin Lakes, NJ, USA) in dark conditions for $30 \mathrm{~min}$. The distribution of cell cycle stages was measured by flow cytometry within $1 \mathrm{~h}$. The cell debris and fixation artifacts were gated out.

\section{Quantitative RT-PCR (Q-PCR)}

Total RNA was extracted from cultured cells using Trizol (Invitrogen, Carlsbad, CA, USA) and reverse transcribed into cDNA using oligo ( $\mathrm{dT}$ ) primers and a cDNA synthesis kit (Invitrogen, USA) according to the manufacturer's protocol. Gene expression was assessed by qRT-PCR using SYBR Premix Dimer Eraser (Perfect Real-Time) assay kits and the following primers:

GAPDH-forward, 5'-GAAGGTGAAGGTCGGAGTC-3';

GAPDH-reverse, 5'-GAAGATGGTGATGGGATTTC-3';

ZNF582-forward 5'-GAGGAGGCGGCAGCTCTACC;

ZNF582-reverse 5'-GAAACGGCAAGACCCAGTGAGAC.

Real-time PCR was performed using the Roche LC480 PCR System and results were analyzed using the comparative $\mathrm{Ct}$ (threshold cycle) method.

\section{Western blotting and immunofluorescence assays}

Crude cellular proteins were separated by SDS-PAGE and transferred to nitrocellulose membranes. Membranes were blocked in 5\% nonfat milk and probed with primary antibodies against ZNF582 (ABclonal, A14453;1:500) p21/Waf (Cell Signaling Technology, 9932kit, 1:1000), p27(KIP1) (Cell Signaling Technology, 9932kit, 1:1000), p21 (Cell Signaling Technology, 9932kit, 1:1000) CyclinD1 (Cell Signaling Technology, 9932kit, 1:1000), CDK2(Cell Signaling Technology, 9932kit, 1:1000),CDK4(Cell Signaling Technology, 9932kit, 1:1000), PI3K (Abcam, ab191606;1: 1000)), Akt (Cell Signaling Technology, 9272,1:1000), and a-tubulin (Abcam, ab7291,1:5000) overnight at $4^{\circ} \mathrm{C}$. Membranes were incubated with horseradish peroxidase (HRP)-conjugated secondary antibodies for $1.5 \mathrm{~h}$ and immunolabeling detected using a Bio-Rad imaging system. Alternatively, fixed cells were hydrated, blocked, incubated with primary antibody against ZNF582 (Biorbyt, 1:200 dilution) and Akt (Cell Signaling Technology, 1:200 dilution) at room temperature for $1 \mathrm{~h}$, incubated with fluorphore-labeled secondary antibody for $10 \mathrm{~min}$, and then examined under fluorescence microscopy.

\section{Animal experiments}


Female BALB/c nude mice (RRID:IMSR_ORNL:BALB/cRI) at 6 weeks of age were obtained from SLA Laboratory Animal (Changsha, China) and housed in a specific pathogen-free facility. Individual mice were subcutaneously injected with $5 \times 10^{6} \mathrm{HeLa}$ cells. Four weeks after inoculation, the mice were sacrificed and the tumors dissected out, weighed, and photographed. The tumor tissues were then fixed in $10 \%$ formalin, paraffin-embedded, sectioned at $3 \mu \mathrm{m}$, and stained with hematoxylin and eosin. The experimental protocol was approved by the Animal Care Committee of Hunan Tumor Hospital and the Affiliated Tumor Hospital of Xiangya Medical College (Changsha, China).

\section{Statistical analysis}

Statistical analyses were performed using SPSS(RRID:SCR_002865)18.0 for Windows (IBM, Inc., Chicago, IL, USA) and PLINK(RRID:SCR_001757)1.07. Continuous variables are presented as mean \pm standard deviation. Qualitative variables are described by frequencies and percentages. The comparison between groups was conducted by $t$-test, ANOVA, Chi-squared test or non-parametric test (Man-Whitney Test) (selected according to the data type) and a p-value of $<0.05$ was considered to indicate significance.

\section{Results}

\section{Radiotherapy reduced ZNF582 methylation level}

The first cohort included 50 patients with locally advanced cervical cancer, and receiving concurrent chemoradiotherapy $(n=37)$ or radiotherapy alone $(n=13)$ (Figure 1$)$, and patients' characteristics are summarized in Table 1. Cervical exfoliated cells were successively collected pre-treatment (0 Gy) and after 24, 30, 36, 48, and 64 Gy doses (Figure 2A). In total, all patients revealed a significant decline in ZNF582 methylation level (ZNF582 ${ }^{\mathrm{m}}$ ) during radiotherapy (Figure 2B). The tendency of ZNF582m level during radiotherapy was consistent with squamous cell carcinoma antigen (SCC-Ag) (Figure 2C)

\section{Higher ZNF582 ${ }^{\mathrm{m}}$ predicted greater radiosensitivity}

In the first group, 22 cases achieved a satisfied response (SR), whereas 28 exhibited modest response (MR) (Figure1); ZNF582 ${ }^{m}$ was significantly higher in the satisfied response group than in the modest response group both in patients receiving radiotherapy only or chemoradiotherapy (Figure $3 A, B, C$ ). In receiver operating characteristic (ROC) curve, a cut off value of 1182 stratified satisfied response and modest response patients with area under the curve (AUC) of $81.01 \%$, sensitivity of $82.14 \%$, and specificity of $72.73 \%$ (Figure 3D).

Among the 50 patients, 21 were defined as high-level ZNF582 methylation (ZNF582 $\left.{ }^{m-h i g h}\right)$ cases and 29 as low-level ZNF582 methylation (ZNF582 ${ }^{m-l o m}$ ) cases according to ROC curve, (Table 2). The ZNF582 ${ }^{m-h i g h}$ patients were more sensitive to radiotherapy than $Z N F 582^{m-l o w}$ patients, both for the entire cohort (76.2\% vs. $20.7 \%$ ) and for patients receiving radiation therapy only (85.7\% vs. $0 \%$ ) (Table 2 ). Representative magnetic resonance images and computed tomography (CT) images of lesions pre- and post-treatment are shown in Figure 3E. 
The above results were confirmed by an independent cohort; $Z N F 582^{m}$ was significantly higher in the satisfied response (SR) group than in the modest response (MR) group, both in the entire cohort ( $n=50$, Figure $4 A)$ and patients receiving radiotherapy only $(n=9$, Figure $4 B)$ or chemoradiotherapy $(n=41$, Figure 4C). ROC curve acquired $84.38 \%$ sensitivity, $83.33 \%$ specificity, and $85.24 \%$ AUC(Figure 4D). The ZNF582 ${ }^{m-h i g h}$ patients were more sensitive to radiotherapy than the ZNF582 ${ }^{m-l o w}$ patients (Table 3). The characteristics of the first cohort and second cohort are presented in Table 4.

\section{ZNF582 ${ }^{\mathrm{m}}$ level change during radiotherapy predicted radiosensitivity the ZNF582 ${ }^{\mathrm{m}-\mathrm{low}}$ group}

A subset of patients (5 of 28 cases) with ZNF582 ${ }^{m-l o w}$ showed a transient increase in $Z N F 582^{m}$ from baseline after receiving 24Gy (representative cases are shown in Figure $5 \mathrm{~A}$ ). This suggests that radiation alone increases $Z N F 582^{m}$, thereby synergistically enhancing radiosensitivity. A subset of patients with ZNF582 ${ }^{m-h i g h}$ demonstrated a rapid decrease in $Z N F 582^{m}$ after $24 \mathrm{~Gy}$, whereas $Z N F 582^{m}$ declined more progressively in others (Figure $5 B$ ). There was no significant difference in radiosensitivity between these two subgroups.

\section{ZNF582 overexpression induced S phase arrest in HeLa cells and increased resistance to radiation}

In our previous study, we found that $Z N F 582^{m}$-negative status was correlated with high ZNF582 protein expression[16] and that ZNF582 overexpression increased the radiation and chemotherapy resistance of cervical cancer cells. To investigate the underlying mechanisms, we transfected ZNF582 into HeLa cells (Figure 6A, B).

ZNF582 overexpressing significantly reduced cell proliferation (Figure 6C), colony formation (Figure 6D) and tumor growth (Figure 6E) of Hela cell. Transcriptome sequencing demonstrated that cell cycle related pathways were significantly up-regulated after ZNF582 overexpression in HeLa cells (Figure 6F). And flow cytometry shown that the proportion of $S$ phase was significantly increased and G1 phase significantly decreased in ZNF582 overexpressing Hela cells (Figure 6H), suggesting that ZNF582 overexpression arrests the cell cycle in the $S$ phase. Further, ZNF582 overexpressing Hela cells demonstrated resistance to radiotherapy than control cells (Figure 6F). Western blotting revealed that transfection of HeLa cells with ZNF582 vector enhanced the expression of p27(KIP1), p21, CDK2, and CDK4, while attenuating the protein expression levels of PI3K, and Akt (Figure 6G); immunohistochemical analysis of tumor tissues in mice also confirmed these phenomena (Figure 6H), suggesting ZNF582 overexpression may induced S phase arrest by stimulating $\mathrm{p} 27(\mathrm{KIP} 1) / \mathrm{p} 21 / \mathrm{CDK} 2 / 4$ signaling in Hela cells. Cell stage is associated with radiation sensitivity (G2/M phase $>\mathrm{G0} / \mathrm{G} 1$ phase $>\mathrm{S}$ phase)[20-24], ZNF582 overexpression may reduce cervical cancer radiosensitivity by arresting the cell cycle in $\mathrm{S}$ phase.

\section{Discussion}

Cervical cancer is one of the most common gynecological malignancies and places a heavy disease burden on low-income and middle-income countries. It is estimated that 94 million cases will increase in 
low- and middle-income countries requiring external beam radiotherapy from 2015 to 2035, of which 70 million will also require treatment with brachytherapy[25]. Chemoradiotherapy (CRT) is the standard treatment for locally advanced cervical cancer[4], and radiotherapy provides the greatest survival benefit for cervical cancer among all malignancies. However, neither radiotherapy nor chemotherapy are effective for a substantial proportion of cases, and the 5-year survival rate remains at only $40-50 \%[26]$. One possible explanation for these unsatisfactory results is the presence of a patient subpopulation with low radiosensitivity at baseline, resulting in reduced clinical efficacy. Therefore, continued efforts are required to improve CRT and identify biomarkers for patients at risk of poor therapeutic response.

Our previous study shown that $Z N F 582^{m}$-positive cervical cancer patients have a better prognosis, but the underlying mechanisms were undefined. Chemoradiotherapy resistance is associated with poor prognosis of cervical cancer, especially in advanced cases, suggesting that ZNF582 ${ }^{m}$ status may regulates chemo- and/or radiosensitivity. In this study, we confirmed that patients with high levels of ZNF582 methylation were more sensitive to radiation therapy (Figures 3, 4 and Tables 2,3) and demonstrated superior clinical outcome. Further, these associations were established non-invasively using exfoliated cervical cells, suggesting that routine $Z N F 582^{m}$ analysis before treatment helps predict the efficacy of radiotherapy for patients with cervical cancer.

SCC-Ag is often used to monitor the therapeutic effect, recurrence, metastasis, and prognosis of cervical cancer[8, 27-29]. Similar to SCC, ZNF582 ${ }^{m}$ was significantly reduced after radiotherapy (Figure2C).

Another major finding of this study was that radiation therapy can trigger an initial transient increase in ZNF582 ${ }^{m}$ among a minority of patients with low basal levels, and these patients showed greater sensitivity than other hypomethylated patients. Further studies are needed to explore predictors and the mechanisms underlying this unusual response to expand the clinical application of radiotherapy. Tumors exhibit numerous anatomical and biological changes during treatment, and these findings support ZNF582 ${ }^{m}$ evaluation during fractionated irradiation as a potentially useful parameter for outcome prediction in addition to baseline parameters. In the entire cohort, there was great variation in the rate of ZNF582 ${ }^{m}$ decline, with a large proportion showing near-zero levels after receiving $24 \mathrm{~Gy}$, while others demonstrated a more gradual decline throughout the entire course (Figure 5B, C). However, there was no significant difference in radiotherapy efficacy between the two groups, possibly owing to the high doses of radiation received.

We also explored the molecular mechanisms of $Z N F 582^{m}$ affecting radiotherapy sensitivity in patients with cervical cancer. Methylation is a negative regulator of gene expression. We reported in our previous study that ZNF582 hypermethylation correlated with low ZNF582 protein expression, similarly, low $Z N F 582^{m}$ correlated with high protein expression[16]. The expression level of ZNF582 was extremely low in HeLa cells (Figure 6A, B). ZNF582 overexpression delayed S/G2-phase in HeLa cells, inducing p27/p21 accumulation and inhibition of the PI3K/Akt pathway, and ZNF582 overexpression increased resistance to radiation (Figure 6). SiHa cells did not show the same phenomenon, which may relate to the origin and 
genetic differences between the two cells. Tumor cells in different cell cycle stages showed different sensitivity to radiation, with highest sensitivity in G2 / M phase, followed by G0 / G1 phase and S phase[21-23]. Accumulation of p27[30-35], p21[36-38], CDK2[39], and CDK4[40] and inhibition of $\mathrm{PI3K} /$ Akt/CyclinD1[41] may contribute to S/G2 cell cycle arrest. and our data suggested that ZNF582 overexpression delayed S/G2-phase in HeLa cells and increased resistance to radiation, these findings may explain why $Z N F 582^{m-l o w}$ patients were more resistant to radiotherapy and demonstrated poorer prognosis[16].

\section{Conclusion}

This study demonstrates that $Z N F 58^{m}$ in exfoliated cervical cells is a reliable biomarker for predicting the early response to radiotherapy and monitoring therapeutic efficacy. As a biomarker, $Z N F 58^{m}$ has several advantages: (1) could be measured in non-invasively exfoliated cells; (2) provides not only a pretreatment predictor of response but also allows therapeutic monitoring during the course of treatment; (3) could be accurately and inexpensively detected with conventional reagents. We conclude that ZNF58m measurement is helpful for early screening, diagnosis, prediction of radiotherapy response, and prognosis of cervical cancer.

\section{Abbreviations}

ZNF582, zinc finger protein 582;ZNF582 ${ }^{m}$, methylated ZNF582 gene; RECIST, Response Evaluation Criteria in Solid Tumors; ROC, receiver operating characteristic; CCRT, concomitant chemoradiotherapy; PBS, phosphate-buffered saline; CR, complete remission; PR, partial response; PD, Progressive disease; SD, Stable disease; M-index, the methylation index; HPV, human papillomavirus; gDNA, Genomic DNA;QMSP, quantitative methylation specific PCR;COL2A, The type Il collagen gene; $\mathrm{Cp}$, The crossing point; SR, Satisfied response; MR, Modest response; AUC, area under the curve; CT, computed tomography; SCC-Ag, Squamous cell carcinoma antigen; CRT, Chemoradiotherapy; FIGO, Federation of Gynecology and Obstetrics; RT, radiation treatment; SCC, Squamous cell carcinoma; SD, standard deviation; ORR, objective remission rate.

\section{Declarations}

\section{Acknowledgments}

The authors thank all the members of Jing Wang laboratory, Hunan Hongya Gene Technology Co., Ltd, and Enago (www.enago.cn) for the English embellishment.

\section{Authors' contributions}

$X Y Z, M C Z, J T C, T H, X T L, C Z Q$ contributed to sample collection. NYW contributed to the writing of this manuscript. XYZ, NYW and MCZ contributed to the clinical data evaluation and the molecular 
experiments. JTC, JW, NYW, QJL contributed to the conception, design, and final approval of the submitted version. JW and NYW provide financial support. XYZ, YW, CF, SYL, and HL contributed to the technical support for the data analysis. All authors read and approved the final manuscript.

\section{Ethics approval and consent to participate}

The study protocol was approved by the Institutional Review Board of Hunan Cancer Hospital and the Animal Ethics Committee of Hunan Cancer Hospital. Informed consent was obtained from all participants in accordance with the Declaration of Helsinki.

\section{Consent for publication Availability of data and material}

The data that support the findings of this study are available on request from the corresponding author. The data are not publicly available due to privacy or ethical restrictions.

\section{Financial support}

This work was supported by the Clinical Research Center in Gynecologic Cancer, Hunan Cancer Hospital, Hunan Province key plan (22018SK2121), the foundation of social development science and technology division (kq2004138,kq1801104), Scientific Research project of Hunan Health Commission (20201487,C2019066), Hunan cancer hospital (QH201908).

\section{Conflicts of Interest}

The authors have no conflicts of interest to declare.

\section{References}

1. Siegel, R.L., K.D. Miller, and A. Jemal, Cancer statistics, 2019. CA Cancer J Clin, 2019. 69(1): p. 7-34.

2. Miller, K.D., et al., Cancer treatment and survivorship statistics, 2019. CA Cancer J Clin, 2019. 69(5): p. 363-385.

3. Bray, F., et al., Global cancer statistics 2018: GLOBOCAN estimates of incidence and mortality worldwide for 36 cancers in 185 countries. CA Cancer J Clin, 2018. 68(6): p. 394-424.

4. Bermudez, A., N. Bhatla, and E. Leung, Cancer of the cervix uteri. Int J Gynaecol Obstet, 2015. 131 Suppl 2: p. S88-95.

5. Wang, S.M. and Y.L. Qiao, Implementation of cervical cancer screening and prevention in Chinachallenges and reality. Jpn J Clin Oncol, 2015. 45(1): p. 7-11.

6. Kokka, F., et al., Hysterectomy with radiotherapy or chemotherapy or both for women with locally advanced cervical cancer. Cochrane Database Syst Rev, 2015(4): p. Cd010260.

7. Yang, J., et al., Effect of radiotherapy on the survival of cervical cancer patients: An analysis based on SEER database. Medicine (Baltimore), 2019. 98(30): p. e16421. 
8. Fu, J., et al., The role of squamous cell carcinoma antigen (SCC Ag) in outcome prediction after concurrent chemoradiotherapy and treatment decisions for patients with cervical cancer. Radiat Oncol, 2019. 14(1): p. 146.

9. Sabulei, C. and J.E. Maree, An exploration into the quality of life of women treated for cervical cancer. Curationis, 2019. 42(1): p. e1-e9.

10. Li, Q., Y. Zhang, and Q. Jiang, SETD3 reduces KLC4 expression to improve the sensitization of cervical cancer cell to radiotherapy. Biochem Biophys Res Commun, 2019. 516(3): p. 619-625.

11. Huang, C., et al., SOX2 regulates radioresistance in cervical cancer via the hedgehog signaling pathway. Gynecol Oncol, 2018. 151(3): p. 533-541.

12. Figueroa, M.E., et al., DNA methylation signatures identify biologically distinct subtypes in acute myeloid leukemia. Cancer Cell, 2010. 17(1): p. 13-27.

13. Huntley, S., et al., A comprehensive catalog of human KRAB-associated zinc finger genes: insights into the evolutionary history of a large family of transcriptional repressors. Genome Res, 2006. 16(5): p. 669-77.

14. Huang, R.L., et al., Methylomic analysis identifies frequent DNA methylation of zinc finger protein 582 (ZNF582) in cervical neoplasms. PLoS One, 2012. 7(7): p. e41060.

15. Liou, Y.L., et al., Combined clinical and genetic testing algorithm for cervical cancer diagnosis. Clin Epigenetics, 2016. 8: p. 66.

16. Wu, N.-Y.Y., et al., High methylation of ZNF582 in cervical adenocarcinoma affects radiosensitivity and prognosis. Annals of Translational Medicine, 2019.

17. Lin, H., et al., Methylated ZNF582 gene as a marker for triage of women with Pap smear reporting low-grade squamous intraepithelial lesions - a Taiwanese Gynecologic Oncology Group (TGOG) study. Gynecol Oncol, 2014. 135(1): p. 64-8.

18. Tian, Y., et al., Utility of gene methylation analysis, cytological examination, and HPV-16/18 genotyping in triage of high-risk human papilloma virus-positive women. Oncotarget, 2017.

19. Liou, Y.L., et al., Comparison of HPV genotyping and methylated ZNF582 as triage for women with equivocal liquid-based cytology results. Clin Epigenetics, 2015. 7: p. 50.

20. Sinclair, W.K. and R.A. Morton, $X$-ray sensitivity during the cell generation cycle of cultured Chinese hamster cells. Radiat Res, 1966. 29(3): p. 450-74.

21. Sinclair, W.K. and R.A. Morton, VARIATIONS IN X-RAY RESPONSE DURING THE DIVISION CYCLE OF PARTIALLY SYNCHRONIZED CHINESE HAMSTER CELLS IN CULTURE. Nature, 1963. 199: p. 1158-60.

22. Terasima, T. and L.J. Tolmach, Variations in several responses of HeLa cells to $x$-irradiation during the division cycle. Biophys J, 1963. 3(1): p. 11-33.

23. Terasima, T. and L.J. Tolmach, $X$-ray sensitivity and DNA synthesis in synchronous populations of HeLa cells. Science, 1963. 140(3566): p. 490-2.

24. Carlson, J.G., X-ray-induced prophase delay and reversion of selected cells in certain avian and mammalian tissues in culture. Radiat Res, 1969. 37(1): p. 15-30. 
25. Rodin, D., et al., Scale-up of radiotherapy for cervical cancer in the era of human papillomavirus vaccination in low-income and middle-income countries: a model-based analysis of need and economic impact. Lancet Oncol, 2019. 20(7): p. 915-923.

26. Wright, J.D., et al., Population-level trends in relative survival for cervical cancer. Am J Obstet Gynecol, 2015. 213(5): p. 670.e1-7.

27. Salvatici, M., et al., Squamous cell carcinoma antigen (SCC-Ag) during follow-up of cervical cancer patients: Role in the early diagnosis of recurrence. Gynecol Oncol, 2016. 142(1): p. 115-119.

28. Wen, Y.F., et al., Elevated circulating tumor cells and squamous cell carcinoma antigen levels predict poor survival for patients with locally advanced cervical cancer treated with radiotherapy. PLoS One, 2018. 13(10): p. e0204334.

29. Shen, Q., et al., Differential Expression of Aquaporins in Cervical Precursor Lesions and Invasive Cervical Cancer. Reprod Sci, 2016. 23(11): p. 1551-1558.

30. Liu, Z., et al., MCM family in HCC: MCM6 indicates adverse tumor features and poor outcomes and promotes S/G2 cell cycle progression. BMC Cancer, 2018. 18(1): p. 200.

31. Wang, A., R. Zeng, and H. Huang, Retinoic acid and sodium butyrate as cell cycle regulators in the treatment of oral squamous carcinoma cells. Oncol Res, 2008. 17(4): p. 175-82.

32. Lin, C.B., C.C. Lin, and G.J. Tsay, 6-Gingerol Inhibits Growth of Colon Cancer Cell LoVo via Induction of G2/M Arrest. Evid Based Complement Alternat Med, 2012. 2012: p. 326096.

33. Sun, X.L., et al., Magnoflorine inhibits human gastric cancer progression by inducing autophagy, apoptosis and cell cycle arrest by JNK activation regulated by ROS. Biomed Pharmacother, 2020. 125: p. 109118.

34. Auld, C.A., K.M. Fernandes, and R.F. Morrison, Skp2-mediated p27(Kip1) degradation during S/G2 phase progression of adipocyte hyperplasia. J Cell Physiol, 2007. 211(1): p. 101-11.

35. Zhong, S., et al., Activation of P27kip 1-cyclin D1/E-CDK2 pathway by polysaccharide from Phellinus linteus leads to S-phase arrest in HT-29 cells. Chem Biol Interact, 2013. 206(2): p. 222-9.

36. Barr, A.R., et al., DNA damage during S-phase mediates the proliferation-quiescence decision in the subsequent $G 1$ via p21 expression. Nat Commun, 2017. 8: p. 14728.

37. Huang, B., et al., Aflatoxin B(1) induces S phase arrest by upregulating the expression of p21 via MYC, PLK1 and PLD1. Biochem Pharmacol, 2019. 166: p. 108-119.

38. Beckerman, R., et al., A role for Chk1 in blocking transcriptional elongation of p21 RNA during the $S$ phase checkpoint. Genes Dev, 2009. 23(11): p. 1364-77.

39. Zhong, S., et al., Protocatechualdehyde Induces S-Phase Arrest and Apoptosis by Stimulating the p27(KIP1)-Cyclin A/D1-CDK2 and Mitochondrial Apoptotic Pathways in HT-29 Cells. Molecules, 2016. 21(7).

40. Ravanko, K., et al., Loss of p27Kip1 from cyclin E/cyclin-dependent kinase (CDK) 2 but not from cyclin D1/CDK4 complexes in cells transformed by polyamine biosynthetic enzymes. Cancer Res, 2000. 60(18): p. 5244-53. 
41. Ahn, H., et al., Antitumor Effect of Pyrogallol via miR-134 Mediated S Phase Arrest and Inhibition of PI3K/AKT/Skp2/cMyc Signaling in Hepatocellular Carcinoma. Int J Mol Sci, 2019. 20(16).

\section{Tables}

Table 1. Characteristics of ZNF582 ${ }^{m-H i g h}$ and ZNF582 ${ }^{\text {m-Low }}$ subjects in the first cohort

\begin{tabular}{|c|c|c|c|c|}
\hline Characteristics & $\begin{array}{l}\text { Patients } \\
(n=50)\end{array}$ & $\begin{array}{l}\text { ZNF582 } \\
H i g h \\
(\mathrm{n}=21)\end{array}$ & $\begin{array}{l}\text { ZNF582m- } \\
\text { Low }(n=29)\end{array}$ & $\begin{array}{l}\mathrm{P} \\
\text { value }\end{array}$ \\
\hline \multicolumn{5}{|l|}{ Specimen } \\
\hline Squamous cell carcinoma & 46 & 20 & 26 & \multirow[t]{3}{*}{0.651} \\
\hline Adenocarcinoma & 1 & 0 & 1 & \\
\hline $\begin{array}{l}\text { Adenosquamous } \\
\text { carcinoma }\end{array}$ & 3 & 1 & 2 & \\
\hline \multicolumn{5}{|l|}{ Age (years) } \\
\hline$<50$ & 9 & 5 & 4 & \multirow[t]{2}{*}{0.363} \\
\hline$\geq 50$ & 41 & 16 & 25 & \\
\hline \multicolumn{5}{|l|}{ FIGO stage } \\
\hline$<\| \mathrm{B}$ & 0 & 0 & 0 & \multirow[t]{2}{*}{1.000} \\
\hline$\geq \mathrm{IIB}$ & 50 & 21 & 29 & \\
\hline \multicolumn{5}{|l|}{ Differentiation } \\
\hline Well/moderate & 33 & 15 & 18 & \multirow[t]{2}{*}{0.288} \\
\hline Poorly & 11 & 3 & 8 & \\
\hline Unknown & 6 & 3 & 3 & \\
\hline \multicolumn{5}{|l|}{ NCRT } \\
\hline With & 37 & 14 & 23 & \multirow[t]{2}{*}{0.314} \\
\hline Without & 13 & 7 & 6 & \\
\hline \multicolumn{5}{|l|}{ LNM } \\
\hline Yes & 34 & 13 & 21 & \multirow[t]{2}{*}{0.432} \\
\hline No & 16 & 8 & 8 & \\
\hline
\end{tabular}


Table 2. Comparison of the efficacy of radiotherapy in first cohort with ZNF582 ${ }^{m-H i g h}$ or ZNF582 ${ }^{m-L o w}$

\begin{tabular}{|c|c|c|c|c|}
\hline & Cases & $\begin{array}{l}\text { Satisfied- } \\
\text { Response }\end{array}$ & $\begin{array}{l}\text { Modest- } \\
\text { Response }\end{array}$ & ORR(\%) \\
\hline \multicolumn{5}{|l|}{ Total } \\
\hline ZNF582 ${ }^{m-H i g h}$ & 21 & 16 & 5 & 76.2 \\
\hline ZNF582 ${ }^{\text {m-Low }}$ & 29 & 6 & 23 & 20.7 \\
\hline$p$-value & & $<0.001^{\star}$ & & \\
\hline \multicolumn{5}{|c|}{ Radiotherapy Alone } \\
\hline ZNF582 ${ }^{m-H i g h}$ & 7 & 6 & 1 & 85.7 \\
\hline ZNF582m-Low & 6 & 0 & 6 & 0 \\
\hline$p$-value & & $0.005^{\star}$ & & \\
\hline \multicolumn{5}{|c|}{ Chemoradiotherapy } \\
\hline ZNF582 ${ }^{m-H i g h}$ & 14 & 10 & 4 & 71.4 \\
\hline ZNF582 $2^{\text {m-Low }}$ & 23 & 6 & 17 & 26.1 \\
\hline$p$-value & & $0.015^{\star}$ & & \\
\hline ORR: Objective & sion Rate & & & \\
\hline
\end{tabular}

Table 3. Comparison of the efficacy of radiotherapy in second cohort with ZNF582 $2^{m-H i g h}$ or ZNF582 ${ }^{m-L o w}$ 


\begin{tabular}{|c|c|c|c|c|}
\hline & Cases & $\begin{array}{l}\text { Satisfied- } \\
\text { Response }\end{array}$ & $\begin{array}{l}\text { Modest- } \\
\text { Response }\end{array}$ & ORR(\%) \\
\hline \multicolumn{5}{|l|}{ Total } \\
\hline ZNF582m-High & 21 & 15 & 6 & 71.43 \\
\hline ZNF582 & 29 & 3 & 26 & 10.34 \\
\hline$p$-value & & $<0.001^{\star}$ & & \\
\hline \multicolumn{5}{|c|}{ Radiotherapy Alone } \\
\hline ZNF582m-High & 2 & 2 & 0 & 100 \\
\hline ZNF582 ${ }^{m-L o w}$ & 7 & 0 & 7 & 0 \\
\hline p-value & & $0.028^{*}$ & & \\
\hline \multicolumn{5}{|c|}{ Chemoradiotherapy } \\
\hline ZNF582 ${ }^{m-H i g h}$ & 19 & 13 & 6 & 68.42 \\
\hline ZNF582 & 22 & 3 & 19 & 13.64 \\
\hline$p$-value & & $<0.001^{\star}$ & & \\
\hline ORR: Objective & Sion Rate & & & \\
\hline
\end{tabular}

Table 4. Characteristics of first cohort and second cohort subjects 


\begin{tabular}{|c|c|c|c|c|}
\hline Characteristics & $\begin{array}{l}\text { Patients } \\
(\mathrm{n}=100)\end{array}$ & $\begin{array}{l}\text { First cohort } \\
(n=50)\end{array}$ & $\begin{array}{l}\text { Second } \\
\text { cohort }(n=50)\end{array}$ & $\begin{array}{l}P \\
\text { value }\end{array}$ \\
\hline \multicolumn{5}{|l|}{ Specimen } \\
\hline Squamous cell carcinoma & 92 & 46 & 46 & \multirow[t]{3}{*}{0.766} \\
\hline Adenocarcinoma & 3 & 1 & 2 & \\
\hline $\begin{array}{l}\text { Adenosquamous } \\
\text { carcinoma }\end{array}$ & 5 & 3 & 2 & \\
\hline \multicolumn{5}{|l|}{ Age (years) } \\
\hline$<50$ & 13 & 9 & 4 & \multirow[t]{2}{*}{0.147} \\
\hline$\geq 50$ & 87 & 41 & 46 & \\
\hline \multicolumn{5}{|l|}{ FIGO stage } \\
\hline$<\| \mathrm{B}$ & 2 & 0 & 2 & \multirow[t]{2}{*}{0.475} \\
\hline$\geq \mathrm{IIB}$ & 98 & 50 & 48 & \\
\hline \multicolumn{5}{|l|}{ Differentiation } \\
\hline Well/moderate & 57 & 33 & 24 & \multirow[t]{2}{*}{0.320} \\
\hline Poorly & 24 & 11 & 13 & \\
\hline Unknown & 19 & 6 & 13 & \\
\hline \multicolumn{5}{|l|}{ NCRT } \\
\hline With & 78 & 37 & 41 & \multirow[t]{2}{*}{0.334} \\
\hline Without & 22 & 13 & 9 & \\
\hline \multicolumn{5}{|l|}{ LNM } \\
\hline Yes & 59 & 34 & 25 & \multirow[t]{2}{*}{0.067} \\
\hline No & 41 & 16 & 25 & \\
\hline
\end{tabular}

\section{Figures}




\section{Explore the relationship between ZNF582 ${ }^{m}$ and radiotherapy sensitivity of cervical cancer patients}

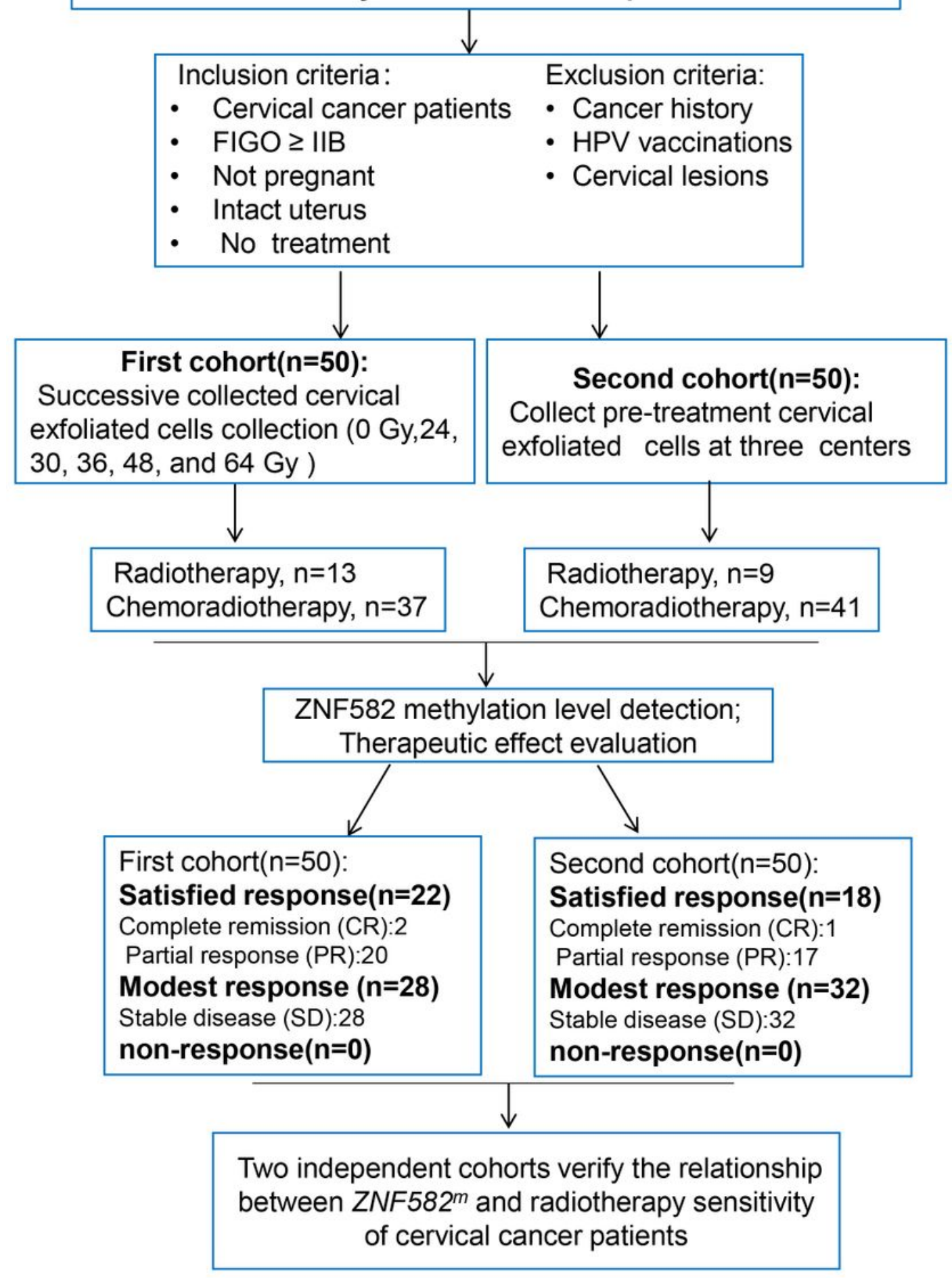

Figure 1

Study design and patient cohort for this study FIGO, Federation of Gynecology and Obstetrics. 


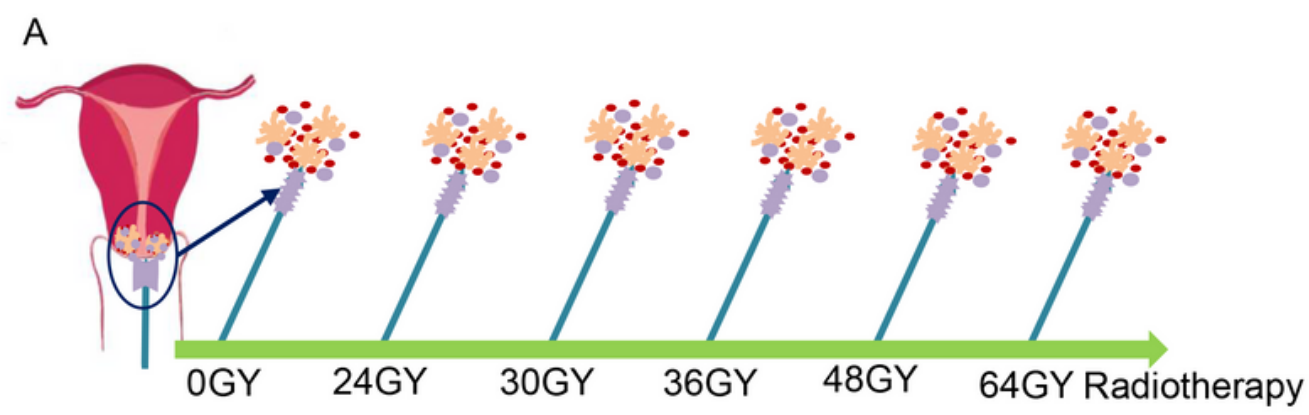

B

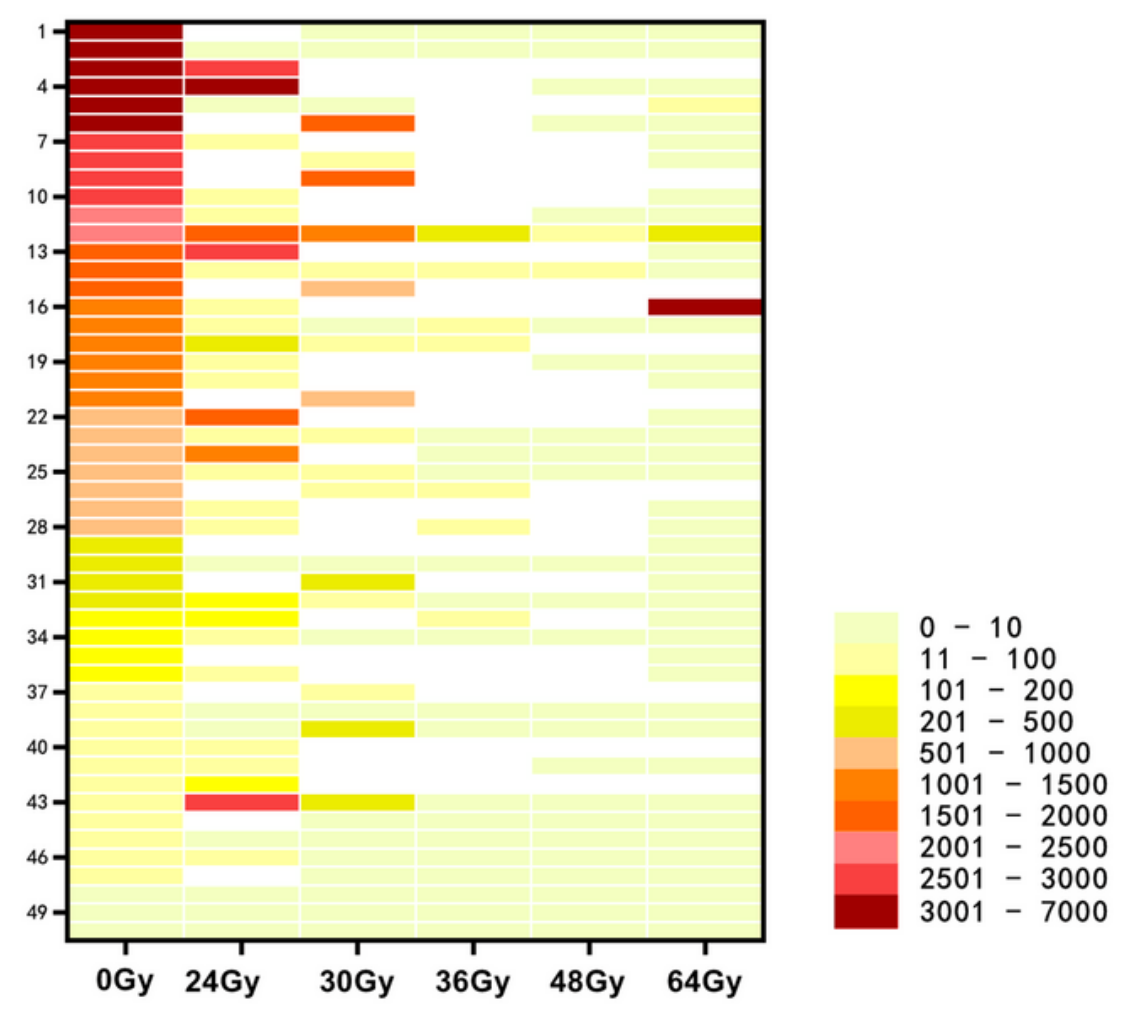

C

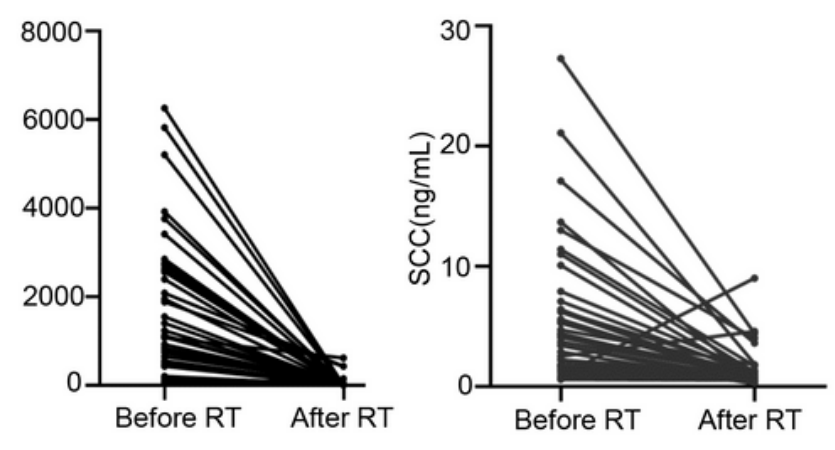

\section{Figure 2}

Changes in ZNF582 methylation level (ZNF582m) during radiotherapy A. Flow chart of specimen collection. Exfoliated cervical cancer cells were collected pre-treatment $(0 \mathrm{~Gy})$ and during treatment (after doses of $24,30,36,48$, and $64 \mathrm{~Gy}$ ). B. Heat map of ZNF582m during radiotherapy (darker color indicates higher ZNF582m). Each row represents one case. C. Both ZNF58m and SCC decreased significantly during radiotherapy. 
A

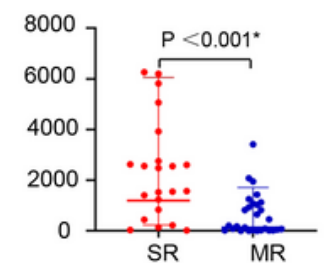

$B$ ह

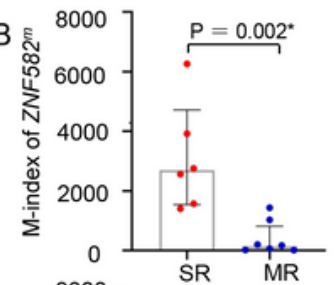

C

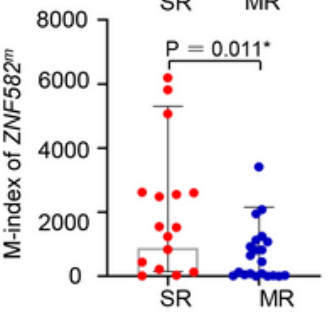

D

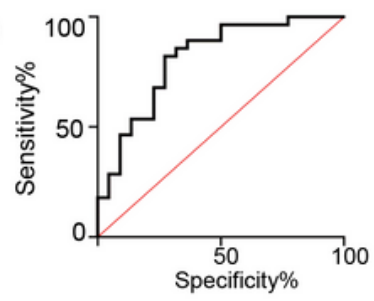

$\mathrm{E}$

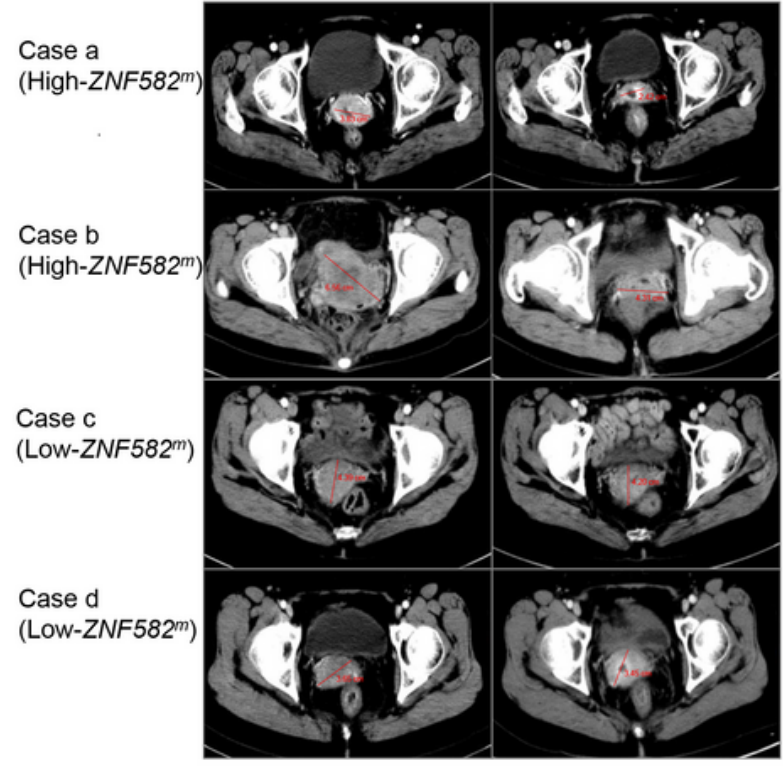

\section{Figure 3}

Difference baseline ZNF582m between patients with satisfied response modest response to radiotherapy. A. Among 50 patients, average baseline ZNF582m (M-index) was significantly greater in the SatisfiedResponse (SR) group than in the Modest-Response(MR) group [2356 \pm 1985 vs. $641 \pm 825, p<0.05^{*}$ by non-parametric test (Mann-Whitney Test)]. B, C. Average ZNF582m values were also higher in the Satisfied-Response (SR) group than in the Modest-Response (MR) group in both chemoradiotherapy 
cases (B) and radiotherapy only cases (C). D. Receiver operating characteristic (ROC) analysis for stratifying SR group from MR group by ZNF582m. The calculated cutoff M-index value of 1182 distinguished SR from MR with $82.14 \%$ sensitivity and $72.73 \%$ specificity. The AUC was $81.01 \%$. E. Representative CT/MR images from ZNF582 m-high and ZNF582 m-low patients before and after radiotherapy.

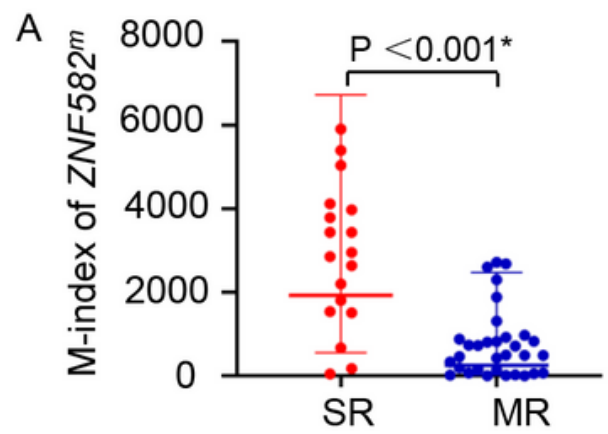

\begin{tabular}{|c|c|c|}
\hline & \multicolumn{2}{|c|}{ ZNF582 ${ }^{m}$ of Total } \\
\hline & $\begin{array}{l}\text { Satisfied- } \\
\text { Response }\end{array}$ & $\begin{array}{l}\text { Modest- } \\
\text { Response }\end{array}$ \\
\hline Case & 18 & 32 \\
\hline Mean $\pm S D$ & $2761 \pm 1712$ & $761 \pm 821$ \\
\hline \multirow[t]{3}{*}{ P-value } & \multicolumn{2}{|c|}{$<0.001^{*}$} \\
\hline & \multicolumn{2}{|c|}{ ZNF582 ${ }^{m}$ of only Radiotherapy } \\
\hline & $\begin{array}{l}\text { Satisfied- } \\
\text { Response }\end{array}$ & $\begin{array}{l}\text { Modest- } \\
\text { Response }\end{array}$ \\
\hline Case & 2 & 7 \\
\hline Mean $\pm S D$ & $4847 \pm 1496$ & $504 \pm 421$ \\
\hline \multirow[t]{3}{*}{ P-value } & \multicolumn{2}{|l|}{$0.04^{*}$} \\
\hline & \multicolumn{2}{|c|}{ ZNF582 ${ }^{m}$ of chemoradiotherapy } \\
\hline & $\begin{array}{l}\text { Satisfied- } \\
\text { Response }\end{array}$ & $\begin{array}{l}\text { Modest- } \\
\text { Response }\end{array}$ \\
\hline Case & 16 & 25 \\
\hline Mean $\pm S D$ & $2613 \pm 1607$ & $833 \pm 895$ \\
\hline \multirow[t]{2}{*}{ P-value } & \multicolumn{2}{|c|}{$<0.001^{*}$} \\
\hline & \multicolumn{2}{|c|}{ ZNF582 } \\
\hline Cutoff-value & \multicolumn{2}{|c|}{1182} \\
\hline Sensitivity & \multicolumn{2}{|c|}{$84.38 \%$} \\
\hline Specificity & \multicolumn{2}{|c|}{$83.33 \%$} \\
\hline AUC & \multicolumn{2}{|c|}{$85.24 \%$} \\
\hline$P$-value & \multicolumn{2}{|c|}{$<0.0001$} \\
\hline
\end{tabular}

Figure 4 
An independent cohort confirmedhighbaseline ZNF582m patients achieving better response than low baseline ZNF582m patients A. Among 50 patients, average baseline ZNF582m (M-index) was significantly greater in the satisfied response (SR) group than in the modest response (MR) group [4847 \pm 1496 vs. $504 \pm 421, \mathrm{p}<0.05^{\star}$ by non-parametric test (Mann-Whitney Test)]. B, C. Average ZNF582m values were also higher in the satisfied response (SR) group than in the modest response (MR) group both in chemoradiotherapy cases (B) and radiotherapy only cases (C). D. Receiver operating characteristic (ROC) analysis for stratifying SR patients from MR patients by ZNF582m. The calculated cutoff M-index value of 1182 distinguished SR from MR with $84.38 \%$ sensitivity and $83.33 \%$ specificity. The AUC was $85.24 \%$. 


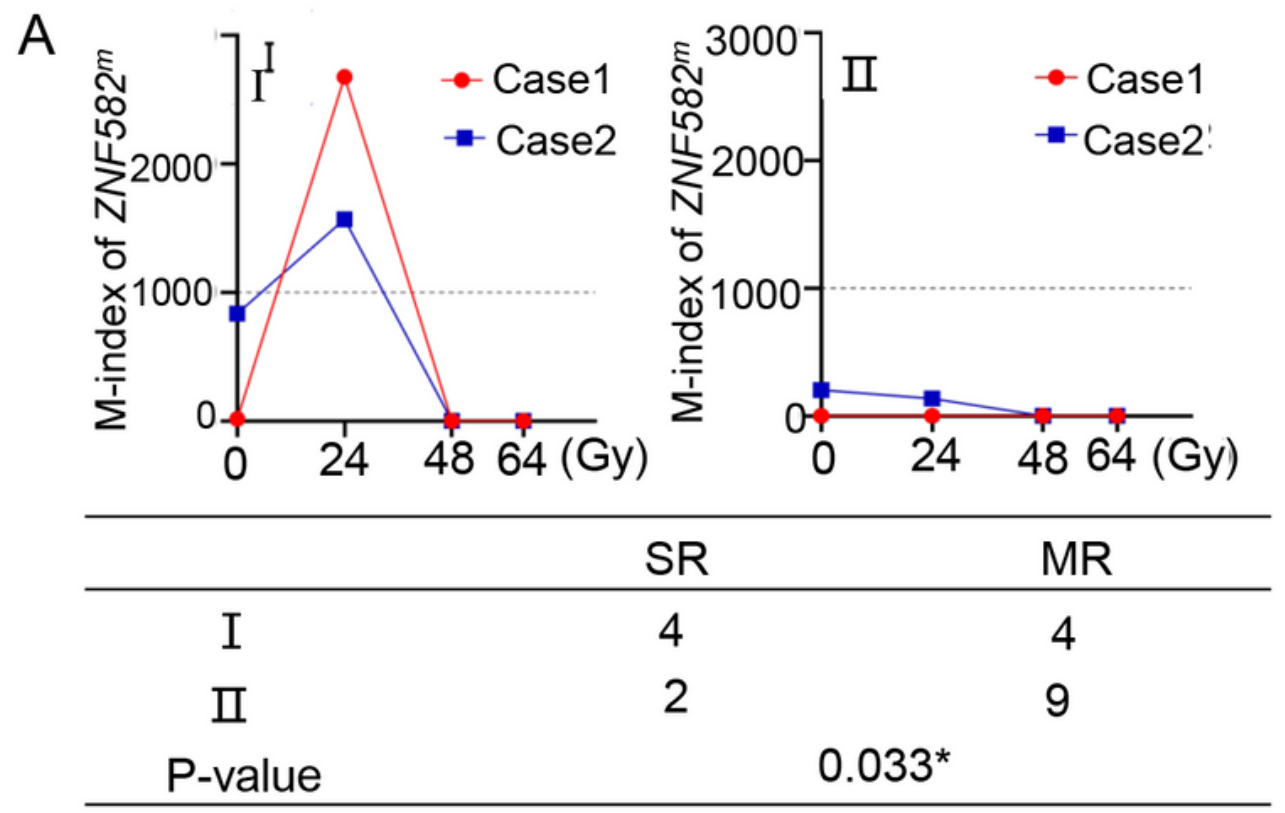

I :ZNF582m-Low increased first and decreased afterwards ㅍ:ZNF582 ${ }^{\text {-Low }}$ not increased

B
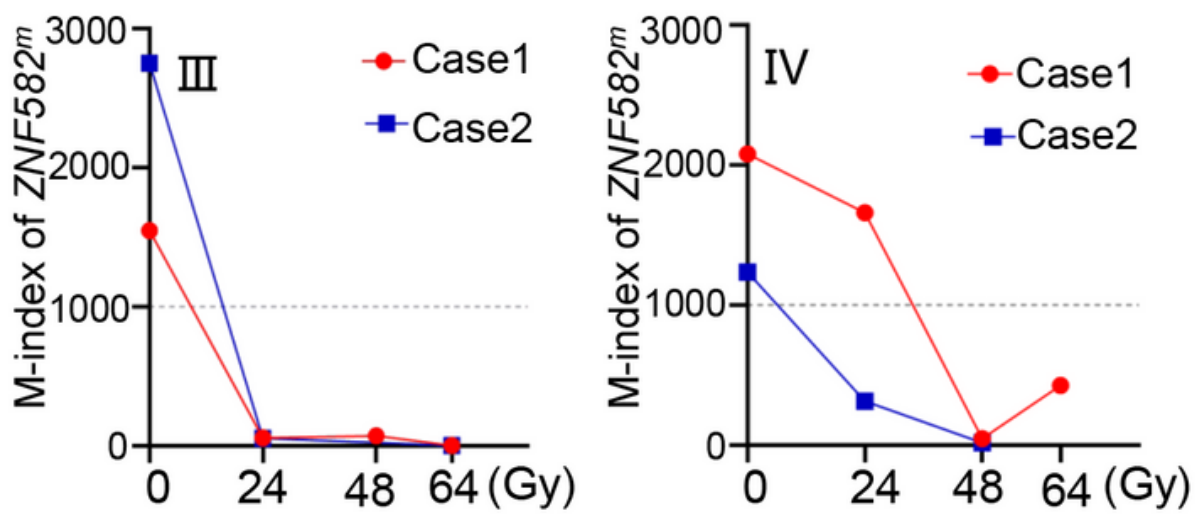

\begin{tabular}{|c|c|c|}
\hline & SR & MR \\
\hline III & 11 & 1 \\
\hline IV & 5 & 4 \\
\hline P-value & & \\
\hline
\end{tabular}

III:ZNF582m-High decreased rapidly IV:ZNF582m-High decreased slowly

Figure 5

Association of the magnitude of ZNF58m change during radiotherapy and therapeutic response A. I) Representative ZNF582m-low cases showing a transient increase in ZNF582m after receiving 24 Gy and then a rapid decline; II) Representative ZNF582m-low cases showing a progressive decline throughout the course of radiotherapy; B. III) Representative ZNF582m-high cases showing a rapid decline after $24 \mathrm{~Gy}$; IV) Representative ZNF58 m-high cases showing a gradual decline during radiotherapy. 
A

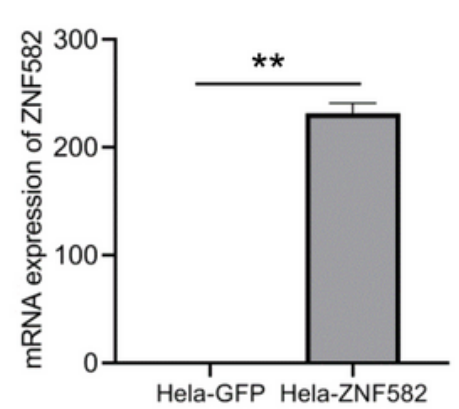

C

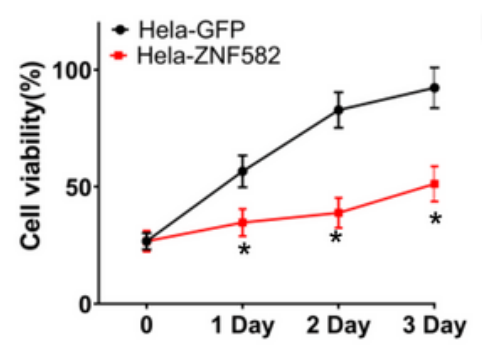

E

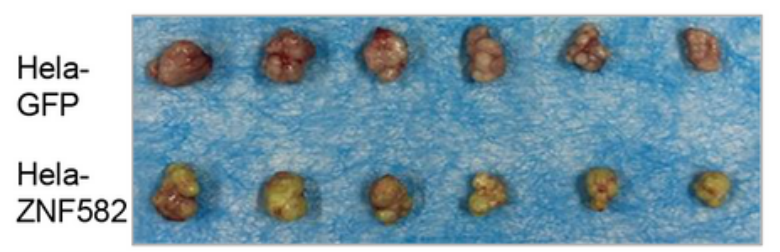

F

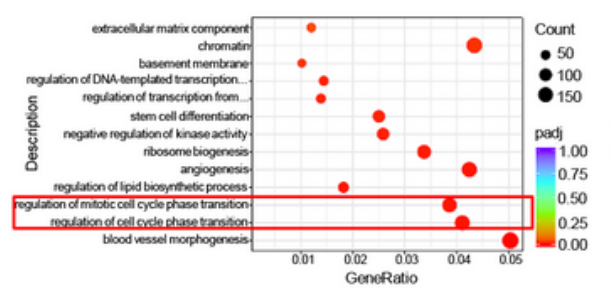

$\mathrm{H}$

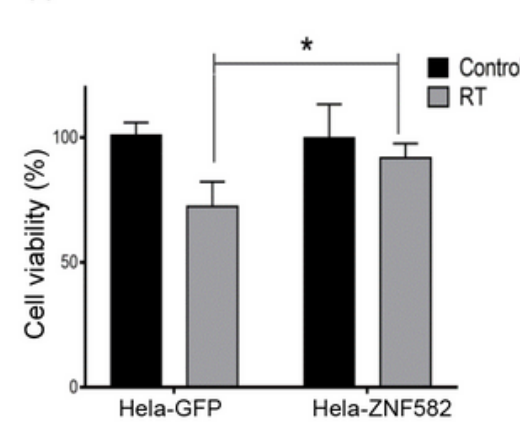

D

G

I
B
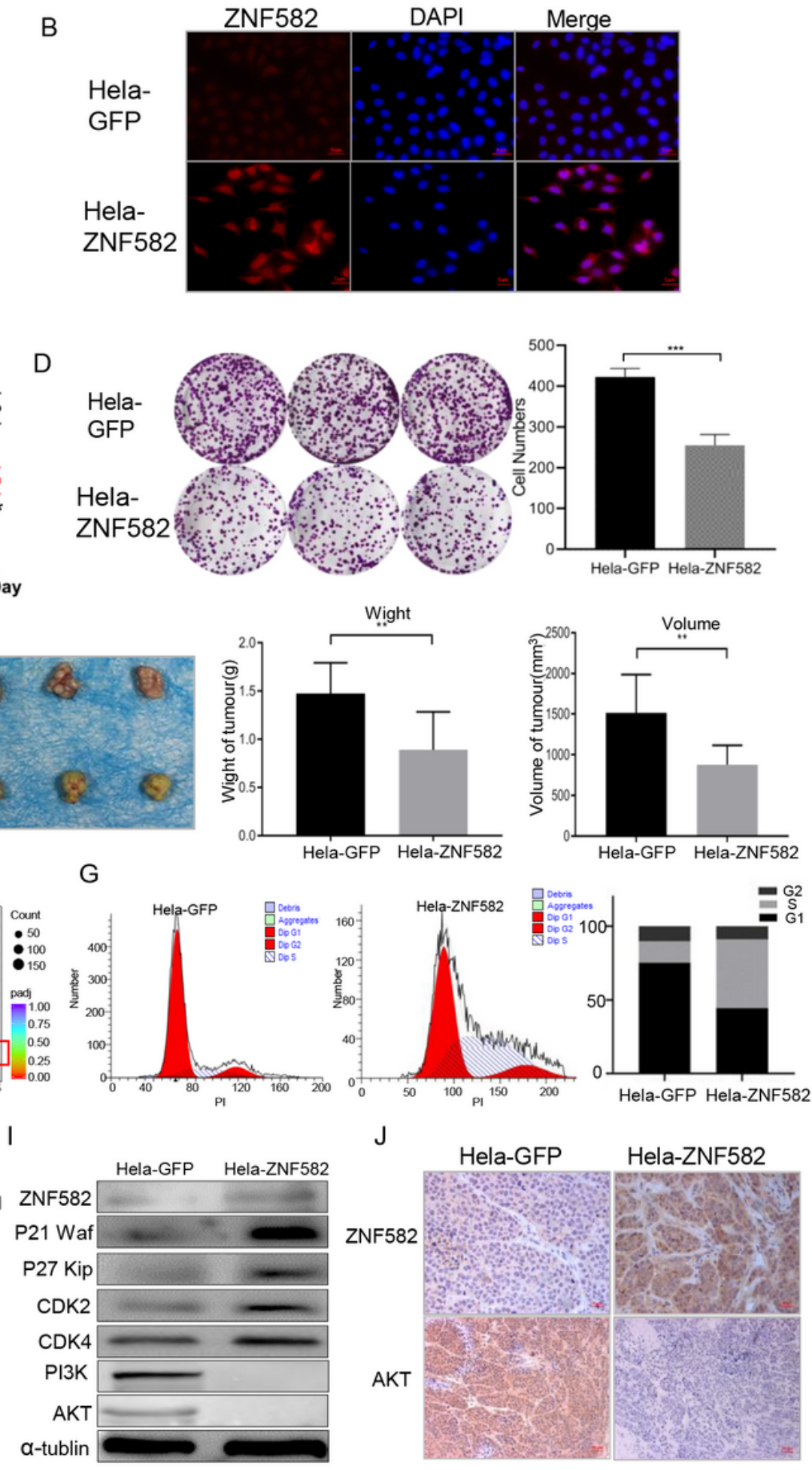

\section{Figure 6}

ZNF582 overexpression induced S phase arrest in Hela cells A-B. Q-PCR and immunocytochemical analyses of ZNF582 mRNA and protein expression in HeLa cells transfected with vector control (GFP) or ZNF582 vector. ZNF582 was overexpressed after transfection with ZNF582 vector compared with the GFP group. C. Cell proliferation rate of Hela-ZNF582 and Hela-GFP; D. Colony formation ability significantly decreased in the HeLa cells with ZNF582 overexpression; E. In the xenografts of HeLa cells 
in nude mice, the tumor volume and weight of the ZNF582-overexpressing cells were significantly lower than those in the control group; F. Bubble chart. GO enrichment analysis of differentially expressed genes of Hela-ZNF582 compare with Hela-GFP; G. Cell cycle stage was detected flow cytometry. H. Viabilities of HeLa cells transfected with ZNF582 or GFP (control) after 6 Gy radiation treatment (RT) for $48 \mathrm{~h}$. I. Cell cycle-associated molecules by HeLa-GFP and HeLa-ZNF582 cells determined by western blotting. J. ZNF582 and Akt expression in the tumor tissues determined by immunohistochemistry. Data are expressed as mean \pm SD of three separate experiments per group (magnification $\times 400$; scale bars $20 \mu \mathrm{m}$; ${ }^{*} p<0.05,{ }^{* \star} p<0.01,{ }^{* \star} \mathrm{p}<0.001$ vs. the controls by $\mathrm{t}$-test. 